\title{
EFFECT OF THE COMPLEX PHYSICAL THERAPY ON THE UPPER EXTREMITY FUNCTION, FUNCTIONAL INDEPENDENCE AND COGNITION IN POST-STROKE PATIENTS
}

\author{
Iriah Uwa-Agbonikhena \\ Department of Neurology and Neurosurgery ${ }^{l}$ \\ iriahuwagbae@gmail.com \\ Viktoriia Gryb \\ Department of Neurology and Neurosurgery \\ gmne@ukr.net \\ Viktoriia Gerasymchuk \\ Department of Neurology and Neurosurgery ${ }^{l}$ \\ viktorianeuro@gmail.com \\ Marta Kupnovytska-Sabadosh \\ Department of Neurology and Neurosurgery ${ }^{l}$ \\ mar.tar.27.12@gmail.com \\ Liubov Maksymchuk \\ Department of Neurology and Neurosurgery \\ lyubamax@gmail.com \\ ${ }^{1}$ Ivano-Frankivsk National Medical University \\ 2 Halytska str., Ivano-Frankivsk, Ukraine, 76018
}

\begin{abstract}
Motor dysfunction and cognitive impairment (CI) are the most prevalent and disabling among the stroke consequences. CI decreases the effectivity of motor rehabilitation, but motor dysfunction itself may also influence the manifestations and progression of CI. So development and study of novel physical therapy tactics, which are aiming to target both of these syndromes, becomes a subject of great interest nowadays.

The aim of study was to evaluate the impact of different physical therapy approaches on the upper extremity function, cognition and functional independence in patients in 1 year after ischemic stroke.

Materials and methods. Totally there were 72 patients examined in the 1-year period after first-ever anterior circulation ischemic stroke. Neurological status, upper extremity function and functional independence were assessed with the National Institutes of Health Stroke Scale (NIHSS), Fugl-Meyer assessment (FMA), modified Rankin Scale (mRS) and the Functional Independence Measurement (FIM). Cognitive function was assessed with the Montreal Cognitive Assessment (MoCA), Frontal Assessment Battery (FAB), Trail-making Test A and B tests (TMT). Patients in Group 1 received secondary stroke prevention therapy and performed the exercises complex for general muscle function improvement for 2 months; patients in Group 2 also performed the exercise complex for paretic hand function improvement for 2 months.

Results. After 2 months of physical therapy a significant increase of the FIM «Self-care», «Transfer» and therefore subtotal motor and total scores was observed in patients in Group 2; in Group 1 significant improvement was observed only in «Transfer» and subtotal motor scores. Adding of the hand exercise to the physical therapy complex (Group 2) appeared to be more beneficial for the upper extremity motor function. Patients in Group 2 after 2 months showed increase of the FMA «Wrist» score by $40 \%(p<0.05)$, «Hand» score by $42.8 \%(p<0.01)$ and «Total motor function» by $30.1 \%(p<0.05)$, and the «Total motor function» score in Group 2 was $10.9 \%$ higher in comparison with the Group $1(p<0.05)$. In cognitive status significant differences compared to baseline level were observed only in Group 2; MoCA score increased by $14.3 \%(p<0.05)$ and TMT-B performance time decreased by $14.8 \%(p<0.05)$. Baseline MoCA score correlated with FMA «Wrist» $(r=0.32 ; p=0.028)$, «Hand» $(r=0.49 ; p=0.001)$ and «Total motor function» $(r=0.46 ; p=0.004)$ scores. TMT-B score showed significant correlation with the FMA «Wrist» $(r=-0.032 ; p=0.025)$, «Hand» $(-0.45 ; p=0.009)$, «Speed/coordination» $(r=-0.023 ; p=0.036)$ and «Total motor function» $(r=-0.42 ; p=0.023)$.

Conclusion. Adding of exercise for hand function improvement into the physical therapy complex for post-stroke patients contributes to better upper extremity motor performance and therefore is more favourable for patients' functional independence.
\end{abstract}


Upper extremity motor impairment, especially hand and wrist dysfunction, are associated with worse cognitive performance. Hand function and fine motor skills improvement could be beneficial for the patients' cognition. Further research is needed in regard to the prognostic significance of these findings and their impact on the treatment and rehabilitation strategies.

Keywords: cognitive impairment, stroke, upper extremity function, physical therapy, functional independence.

DOI: $10.21303 / 2504-5679.2021 .001768$

\section{Introduction}

Stroke remains one of the leading causes of disability nowadays. A number of disabled stroke survivors is constantly rising, mostly due to its high incidence and a significant decrease in stroke mortality within the recent decades [1].

Motor and cognitive dysfunction are the most prevalent and disabling among the stroke consequences and have a high impact life quality of the patients as well as their caregivers [2, 3]. Cognitive impairment (CI) and especially dementia decrease the effectivity of motor rehabilitation [4]. Moreover, recent studies determined that motor function may also influence the course, manifestations and progression of CI. Gait speed and grip strength may be risk factors of cognitive decline in elderly patients [5]. Manual dexterity and fine motor skills are considered one of the most important predictors influencing the further CI in patients with cardiovascular diseases [6, 7]. Probably these interactions are associated with the major role of the frontal lobe in cognition, especially executive functions, and motor control [8], so fine motor skills and manual dexterity improvement may also be beneficial for cognition. There is similar evidence about the favourable influence of general physical activity on cognitive functioning of patients with mild CI and dementia $[9,10]$. Benefits of general physical activity for post-stroke patients have also been reported [11-13], though they have not focused on precise evaluation and improvement of hand motor function.

Although the most active and effective recovery takes place within the first 3 months after stroke [14], rehabilitation in later periods becomes even more relevant due to delay or ineffective in the early rehabilitation period. That is why research, development and implementation of novel physical therapy tactics becomes a subject of great interest and creates a prospective of increase of rehabilitation efficacy.

The aim of study was to evaluate the impact of different physical therapy approaches on the upper extremity function, cognition and functional independence in patients in 1 year after ischemic stroke.

\section{Materials and methods}

The study was performed in the department of neurology and neurosurgery of Ivano-Frankivsk National Medical University and the vascular neurology department of Ivano-Frankivsk Regional Clinical Hospital in 2018-2020.

The study design was assessed and approved by the Ethics Commission of Ivano-Frankivsk National Medical University (Protocol No. 101/18, 12.04.2018). All experiments involving participants were conducted in accordance with the World Medical Association Declaration of Helsinki. All patients have signed an informed consent before involvement into the study.

Totally there were 72 patients (47 males, 25 females; mean age 62 [53; 72] years) examined in the 1-year period after first-ever anterior circulation ischemic stroke. Stroke was localized in the left cerebral hemisphere in 39 (54.2\%) patients and in the right hemisphere in $33(45.8 \%)$ patients.

Exclusion criteria:

1. Haemorrhagic stroke;

2. Recurrent stroke;

3. Posterior circulation stroke;

4. Age $<50$ and $>80$;

5. Left-handed patients;

6. Period of $<12$ month and $>24$ month after stroke;

7. Plegia or severe paresis of the upper extremity;

8. modified Rankin Scale (mRS) score $>2$; 
9. Sensory or severe motor aphasia;

10. Malignancy and systemic comorbidities;

11. Severe depression (Hospital Anxiety and Depression Scale (HADS) score >11);

12. previous disability (orthopaedic, etc).

NeurologicalstatuswasassessedwiththeNationalInstitutesofHealthStrokeScale(NIHSS) [15], examination of the upper extremity function was performed with the Fugl-Meyer assessment (FMA) [16]. Functional independence was evaluated with the mRS [17] and the Functional Independence Measurement (FIM) [18]. Cognitive function was assessed with the Montreal Cognitive Assessment (MoCA) [19], Frontal Assessment Battery (FAB) [20], Trail-making Test A and B (TMT) [21]. Screening for depression was performed with the help of HADS scale [22]; pre-existing CI was excluded with the IQCODE questionnaire [23].

Patients were randomized into 2 groups. Patients in Group $1(n=35)$ received secondary stroke prevention therapy and performed the exercise complex for improvement of general muscle function and mobility; patients in Group $2(n=37)$ received secondary stroke prevention therapy, performed the exercise complex for improvement of general muscle function and mobility and also complex of exercises for improvement of the paretic hand function for 2 months. Patients were examined before and after 2 months of the abovementioned therapy.

Statistical analysis of the results was carried out with the help «MS Excel» and a software environment for statistical calculations «R» (R Core Team RR.) using nonparametric evaluation methods. Data are shown as Median (Me) and interquartile interval [Q25 \%; Q75 \%]. Correlation between the parameters was estimated with the Spearman's rank correlation coefficient. Statistical significance was defined as p-value $<0.05$.

\section{Results}

Examination of the patients' baseline neurological status and functional independence (Table 1) showed decreased FIM total and motor subtotal scores due to poor performance in the «Self-care», «Transfer» and «Locomotion» subtests. After 2 months of physical therapy a significant increase of the «Self-care», «Transfer» and therefore subtotal motor and total scores was observed in patients in Group 2; in Group 1 significant improvement was observed only in «Transfer» and subtotal motor scores.

Table 1

Dynamics of the functional independence and neurological status of post-stroke patients, Me [Q25\%; Q75 \%]

\begin{tabular}{ccccc}
\hline & \multicolumn{3}{c}{ Score } \\
\cline { 2 - 5 } Parameter & \multicolumn{3}{c}{ Group 1 } & Group 2 \\
\cline { 2 - 5 } & Baseline & After 2 months & Baseline & After 2 months \\
\hline NIHSS & $8[6 ; 10]$ & $6[4 ; 8]$ & $8[5 ; 10]$ & $6[4 ; 9]$ \\
mRS & $2[1 ; 2]$ & $1[1 ; 2]$ & $2[1 ; 2]$ & $1[1 ; 2]$ \\
\hline Self-care $(0-42)$ & & FIM parameters (range) & $33[28 ; 38]^{*}$ \\
Sphincter control $(0-14)$ & $13[11 ; 14]$ & $29[24 ; 35]$ & $26[20 ; 33]$ & $13[12 ; 14]$ \\
Transfer $(0-21)$ & $14[12 ; 17]$ & $13[12 ; 14]$ & $13[11 ; 14]$ & $18[16 ; 19]^{*}$ \\
Locomotion $(0-14)$ & $9[7 ; 12]$ & $17[14 ; 19]^{*}$ & $14[12 ; 16]$ & $11[10 ; 12]$ \\
Motor subtotal score $(0-91)$ & $63[51 ; 78]$ & $11[9 ; 12]$ & $10[7 ; 12]$ & $75[66 ; 83]^{*}$ \\
Communication $(0-14)$ & $11[10 ; 13]$ & $10[59 ; 80]^{*}$ & $63[50 ; 75]$ & $12[11 ; 13]$ \\
Social cognition $(0-21)$ & $14[11 ; 18]$ & $14[11 ; 18]$ & $11[10 ; 13]$ & $15[12 ; 18]$
\end{tabular}

Note: *-significant difference from the baseline level $(p<0.05)$ 
Examination of patients' baseline upper extremity function with the FMA detected motor dysfunction, which mostly manifested in low «Wrist» and «Hand» subtests scores and therefore resulted in decreased «Total motor function» (Table 2). 2 months of physical therapy for general muscle function and mobility improvement (Group 1) caused increase of the «Total motor function» FMA score by $15.0 \%(p<0.05)$. Adding of the hand exercise to the physical therapy complex (Group 2) appeared to be more beneficial for the upper extremity motor function. Patients in Group 2 after 2 months showed increase of the «Wrist» score by $40 \%(p<0.05)$, «Hand» score by $42.8 \%(p<0.01)$ and «Total motor function» by $30.1 \%(p<0.05)$. Moreover, the «Total motor function» score in Group 2 was $10.9 \%$ higher in comparison with the Group $1(p<0.05)$.

Table 2

Dynamics of the upper extremity function in post-stroke patients, Me [Q25 \%; Q75 \%]

\begin{tabular}{|c|c|c|c|c|}
\hline \multirow{3}{*}{ FMA parameter (range) } & \multicolumn{4}{|c|}{ Score } \\
\hline & \multicolumn{2}{|c|}{ Group 1} & \multicolumn{2}{|c|}{ Group 2} \\
\hline & Baseline & After 2 months & Baseline & After 2 months \\
\hline Upper extremity (0-36) & $24[17 ; 30]$ & $27[21 ; 32]$ & $24[18 ; 32]$ & $29[23 ; 34]$ \\
\hline Wrist (0-10) & $5[3 ; 7]$ & $6[4 ; 8]$ & $5[2 ; 7]$ & $7[5 ; 9]^{*}$ \\
\hline Hand (0-14) & $8[5 ; 10]$ & $9[6 ; 11]$ & $7[4 ; 9]$ & $10[7 ; 12]^{* *}$ \\
\hline Speed/coordination (0-6) & $4[3 ; 5]$ & $4[4 ; 5]$ & $4[3 ; 5]$ & $5[4 ; 5]$ \\
\hline Total motor function $(0-66)$ & $40[27 ; 52]$ & $46[34 ; 57]^{*}$ & $39[26 ; 53]$ & $51[39 ; 60]^{* / * * *}$ \\
\hline Sensation $(0-12)$ & $8[5 ; 10]$ & $9[6 ; 11]$ & $8[6 ; 10]$ & $10[5 ; 10]$ \\
\hline Passive joint movement (0-24) & $14[11 ; 20]$ & $14[11 ; 22]$ & $13[10 ; 19]$ & $14[10 ; 20]$ \\
\hline Joint pain (0-24) & $17[16 ; 20]$ & $18[16 ; 21]$ & $18[16 ; 21]$ & $18[16 ; 21]$ \\
\hline
\end{tabular}

Note: * - significant difference from the baseline level $(p<0.05)$; ** - significant difference from the baseline level $(p<0.01)$; *** - significant difference from the Group 1 level $(p<0.05)$

Evaluation of cognitive functions determined decreased total MoCA and FAB tests score compared to cut-off levels, and increase of TMT-A and TMT-B performance time (Table 3). After 2 months of physical therapy significant differences compared to baseline level were observed only in Group 2; MoCA score increased by $14.3 \%(p<0.05)$ and TMT-B performance time decreased by $14.8 \%(p<0.05)$.

Table 3

Dynamics of the cognitive functions parameters in post-stroke patients, Me [Q25 \%; Q75 \%]

\begin{tabular}{|c|c|c|c|c|}
\hline \multirow{3}{*}{ Parameter (range) } & \multicolumn{4}{|c|}{ Score } \\
\hline & \multicolumn{2}{|c|}{ Group 1} & \multicolumn{2}{|c|}{ Group 2} \\
\hline & Baseline & After 2 months & Baseline & After 2 months \\
\hline $\operatorname{MoCA}(0-30)$ & $22[18 ; 24]$ & $23[19 ; 25]$ & $21[17 ; 24]$ & $24[19 ; 24]^{*}$ \\
\hline FAB $(0-18)$ & $13[12 ; 14]$ & $13[12 ; 14]$ & $13[12 ; 15]$ & $14[12 ; 14]$ \\
\hline TMT-A (sec) & $54[45 ; 70]$ & $50[43 ; 65]$ & $53[44 ; 69]$ & $49[41 ; 62]$ \\
\hline TMT-B (sec) & $146[125 ; 167]$ & $138[121 ; 159]$ & $149[129 ; 176]$ & $127[109 ; 147]^{*}$ \\
\hline
\end{tabular}

Note: *-significant difference from the baseline level $(p<0.05)$ 
Assessment of correlations between the baseline cognitive and FMA motor parameters was performed for determination of the associations between these indices. The most significant correlations were found out between the FMA «Hand», «Total motor function» subtests and cognitive functions indices (Table 4). MoCA score correlated with FMA «Wrist» $(r=0.32 ; p=0.028)$, «Hand» $(r=0.49 ; p=0.001)$ and «Total motor function» $(r=0.46 ; p=0.004)$ scores. TMT-B score showed significant correlation with the FMA «Wrist» $(r=-0.032 ; p=0.025)$, «Hand» $(r=-0.45$; $p=0.009)$, «Speed/coordination» $(r=-0.023 ; p=0.036)$ and «Total motor function» $(r=-0.42$; $p=0.023)$. «Hand» score also correlated with the FAB $(r=0.41 ; p=0.022)$ and TMT-A $(r=-0.29$; $p=0.037)$ scores.

\section{Table 4}

Correlations between the arm motor function and cognition parameters in post-stroke patients

\begin{tabular}{|c|c|c|}
\hline FMA parameter & $r$ & $p$-value \\
\hline \multicolumn{3}{|c|}{ MoCA } \\
\hline Upper extremity & 0.19 & 0.114 \\
\hline Wrist & $0.32 *$ & 0.028 \\
\hline Hand & $0.49 *$ & 0.001 \\
\hline Speed/coordination & 0.37 & 0.075 \\
\hline Total motor function & $0.46^{*}$ & 0.004 \\
\hline \multicolumn{3}{|c|}{ FAB } \\
\hline Upper extremity & 0.19 & 0.119 \\
\hline Wrist & 0.37 & 0.096 \\
\hline Hand & $0.41^{*}$ & 0.022 \\
\hline Speed/coordination & 0.23 & 0.097 \\
\hline Total motor function & $0.43^{*}$ & 0.015 \\
\hline \multicolumn{3}{|c|}{ TMT-A } \\
\hline Upper extremity & -0.37 & 0.203 \\
\hline Wrist & -0.41 & 0.106 \\
\hline Hand & $-0.29^{*}$ & 0.037 \\
\hline Speed/coordination & -0.16 & 0.099 \\
\hline Total motor function & -0.34 & 0.067 \\
\hline \multicolumn{3}{|c|}{ TMT-B } \\
\hline Upper extremity & -0.22 & 0.094 \\
\hline Wrist & $-0.32 *$ & 0.025 \\
\hline Hand & $-0.45^{*}$ & 0.009 \\
\hline Speed/coordination & $-0.23^{*}$ & 0.036 \\
\hline Total motor function & $-0.42^{*}$ & 0.023 \\
\hline
\end{tabular}

Note: *-significance of correlation 


\section{Discussion}

Interactions between cognitive and motor impairment attract more attention during the last years. Prevalence of post-stroke CI is high and varies from 19 to $92 \%$, depending on severity and the method used for its detection [2, 3, 24]. Upper extremity motor impairment is a leading factor that impacts long-term disability due to a major role of manual dexterity in patients' self-care and functional independence. These syndromes are usually studied and treated as distinct entities, though both of them may influence each other's course and manifestations.

A significant improvement of the FIM total and motor subtotal scores in Group 2 patients, who performed physical exercise for improvement of both general muscle function and hand function, was mostly due to increase of the «Self-care» subtest score. This subtest assesses activities of eating, grooming, bathing and toileting, which are provided by the upper extremity. Patients of both groups demonstrated increase of FMA upper extremity «Total motor function» score in comparison with the baseline level, though this index was significantly higher in Group 2 because of hand and wrist function parameters. Total FIM score after 2 months significantly increased from the baseline level only in Group 2. This fact allows us to assume that physical therapy which is targeting upper extremity function is more beneficial for patients' functional independence.

There is evidence that handgrip weakness and asymmetry are associated with CI [6, 25] and hand motor dysfunction may be considered a probable predictor of further cognitive decline in the cardiovascular patients' population [7]. In our study the most significant correlations were observed between the baseline arm motor functioning and cognitive scales which include examination of the executive functions (TMT A and B, FAB, MoCA). Fine motor skills impairment, which resulted in decreased FMA «Hand», «Wrist» and «Total motor function» scores in examined patients also appeared to be associated with the CI manifestations; upper arm functioning score didn't demonstrate significant correlation with cognition.

Positive dynamics of cognitive functioning was observed in Group 2 in MoCA score and TMT-B performance time. These findings may be explained by the role of the frontal lobe in providing of both motor control, cognition and executive functions in particular, which is the underlying basis of these interactions between cognition and fine motor skills [8].

Finally, these findings suggest that inclusion of exercise which precisely target hand function and fine motor skills into their physical therapy complex favourably influences their functional independence and may also be beneficial for cognition.

Study limitations. The study involved a small number of patients. Future studies will benefit from increase of the number of patients and a longer period of physical therapy interventions.

Prospects for further research. Further research with inclusion of larger number of patients, assessment of their motor and cognitive status dynamics after a longer period of time is needed in regard to the prognostic significance of the interactions between the hand functioning and cognition and their impact on the treatment and rehabilitation strategies of post-stroke patients.

\section{Conclusions}

1. Adding of exercise for hand function improvement into the physical therapy complex for post-stroke patients contributes to better upper extremity motor performance (increase of «Wrist» score by $40.0 \%(p<0.05)$, «Hand» score by $42.8 \%(p<0.01)$ and «Total motor function» by $30.1 \%(p<0.05)$ from baseline and therefore is more favourable for patients' functional independence.

2. Upper extremity motor impairment, especially hand and wrist dysfunction, are associated with worse cognitive performance in $\operatorname{MoCA}(r=0.49 ; p=0.001 ; r=0.32 ; p=0.028$, respectively) and TMT-B ( $r=-0.45 ; p=0.009 ; r=-0.32 ; p=0.025$, respectively) tests.

3. Hand function and fine motor skills improvement could be beneficial for the patients' cognition.

\section{Conflict of interests}

The authors declare that they have no conflicts of interest. 


\section{References}

[1] Krishnamurthi, R. V., Moran, A. E., Feigin, V. L., Barker-Collo, S., Norrving, B. et. al. (2015). Stroke Prevalence, Mortality and Disability-Adjusted Life Years in Adults Aged 20-64 Years in 1990-2013: Data from the Global Burden of Disease 2013 Study. Neuroepidemiology, 45 (3), 190-202. doi: http://doi.org/10.1159/000441098

[2] Coco, D., Lopez, G., Corrao, S. (2016). Cognitive impairment and stroke in elderly patients. Vascular Health and Risk Management, 12, 101-105. doi: http://doi.org/10.2147/vhrm.s75306

[3] Brainin, M., Tuomilehto, J., Heiss, W.-D., Bornstein, N. M., Bath, P. M. W. et. al. (2014). Post-stroke cognitive decline: an update and perspectives for clinical research. European Journal of Neurology, 22 (2), 229-e16. doi: http://doi.org/10.1111/ene.12626

[4] Mullick, A. A., Subramanian, S. K., Levin, M. F. (2015). Emerging evidence of the association between cognitive deficits and arm motor recovery after stroke: A meta-analysis. Restorative Neurology and Neuroscience, 33 (3), 389-403. doi: http:// doi.org/10.3233/rnn-150510

[5] Ruan, Y., Shi, Y., Guo, Y. F., Sun, S. Y., Huang, Z. Z. et. al. (2020). Association between grip strength, rapid gait speed and cognition in people aged 50 and above in Shanghai during 2009-2010. Zhonghua Yu Fang Yi Xue Za Zhi, 54 (12), $1414-1420$. doi: http://doi.org/10.3760/cma.j.cn112150-20200714-01003

[6] McGrath, R., Cawthon, P. M., Cesari, M., Al Snih, S., Clark, B. C. (2020). Handgrip Strength Asymmetry and Weakness Are Associated with Lower Cognitive Function: A Panel Study. Journal of the American Geriatrics Society, 68 (9), $2051-2058$. doi: http://doi.org/10.1111/jgs.16556

[7] Ishihara, K., Izawa, K. P., Kitamura, M., Shimogai, T., Kanejima, Y., Morisawa, T., Shimizu, I. (2020). Pinch strength is associated with the prevalence of mild cognitive impairment in patients with cardiovascular disease. Journal of Cardiology, 75 (6), 594-599. doi: http://doi.org/10.1016/j.jjcc.2019.12.009

[8] Leisman, G., Moustafa, A., Shafir, T. (2016). Thinking, Walking, Talking: Integratory Motor and Cognitive Brain Function. Frontiers in Public Health, 4. doi: http://doi.org/10.3389/fpubh.2016.00094

[9] Law, C.-K., Lam, F. M., Chung, R. C., Pang, M. Y. (2020). Physical exercise attenuates cognitive decline and reduces behavioural problems in people with mild cognitive impairment and dementia: a systematic review. Journal of Physiotherapy, 66 (1), 9-18. doi: http://doi.org/10.1016/j.jphys.2019.11.014

[10] Biazus-Sehn, L. F., Schuch, F. B., Firth, J., Stigger, F. de S. (2020). Effects of physical exercise on cognitive function of older adults with mild cognitive impairment: A systematic review and meta-analysis. Archives of Gerontology and Geriatrics, 89 , 104048. doi: http://doi.org/10.1016/j.archger.2020.104048

[11] Han, P., Zhang, W., Kang, L., Ma, Y., Fu, L. et. al. (2017). Clinical Evidence of Exercise Benefits for Stroke. Advances in Experimental Medicine and Biology, 1000, 131-151. doi: http://doi.org/10.1007/978-981-10-4304-8_9

[12] Debreceni-Nagy, A., Horváth, J., Bajuszné Kovács, N., Fülöp, P., Jenei, Z. (2019). The effect of low-intensity aerobic training on cognitive functions of severely deconditioned subacute and chronic stroke patients: a randomized, controlled pilot study. International Journal of Rehabilitation Research, 42 (3), 275-279. doi: http://oi.org/10.1097/mrr.0000000000000346

[13] Blanchet, S., Richards, C. L., Leblond, J., Olivier, C., Maltais, D. B. (2016). Cardiorespiratory fitness and cognitive functioning following short-term interventions in chronic stroke survivors with cognitive impairment: a pilot study. International Journal of Rehabilitation Research, 39 (2), 153-159. doi: http://doi.org/10.1097/mrr.0000000000000161

[14] Hara, Y. (2015). Brain Plasticity and Rehabilitation in Stroke Patients. Journal of Nippon Medical School, 82 (1), 4-13. doi: http://doi.org/10.1272/jnms.82.4

[15] Brott, T., Adams, H. P., Olinger, C. P., Marler, J. R., Barsan, W. G., Biller, J. et. al. (1989). Measurements of acute cerebral infarction: a clinical examination scale. Stroke, 20 (7), 864-870. doi: http://doi.org/10.1161/01.str.20.7.864

[16] Fugl-Meyer, A. R., Jaasko, L., Leyman, I., Olsson, S., Steglind, S. (1975). The post-stroke hemiplegic patient. A method for evaluation of physical performance. Scandinavian Journal of Rehabilitation Medicine, 7, 13-31.

[17] Rankin, J. (1957). Cerebral Vascular Accidents in Patients over the Age of 60: II. Prognosis. Scottish Medical Journal, 2 (5), 200-215. doi: http://doi.org/10.1177/003693305700200504

[18] McDowell, I., Newell, C. (1996). Measuring health: a guide to rating scales and questionnaires. New York: Oxford University Press, 63-67.

[19] Nasreddine, Z. S., Phillips, N. A., Badirian, V., Charbonneau, S., Whitehead, V., Collin, I. et. al. (2005). The Montreal Cognitive Assessment, MoCA: A Brief Screening Tool For Mild Cognitive Impairment. Journal of the American Geriatrics Society, 53 (4), 695-699. doi: http://doi.org/10.1111/j.1532-5415.2005.53221.x

[20] Dubois, B., Slachevsky, A., Litvan, I., Pillon, B. (2000). The FAB: A frontal assessment battery at bedside. Neurology, 55 (11), 1621-1626. doi: http://doi.org/10.1212/wnl.55.11.1621

[21] Corrigan, J. Hinkeldey, N. (1987). Relationships between Parts A and B of the Trail Making Test. Journal of Clinical Psychology, 43 (4), 402-409. doi: http://doi.org/10.1002/1097-4679(198707)43:4<402::aid-jclp2270430411>3.0.co;2-e 
[22] Zigmond, A. S., Snaith, R. P. (1983). The Hospital Anxiety and Depression Scale. Acta Psychiatrica Scandinavica, 67 (6), 361-370. doi: http://doi.org/10.1111/j.1600-0447.1983.tb09716.x

[23] Jorm, A. F. (1994). A short form of the Informant Questionnaire on Cognitive Decline in the Elderly (IQCODE): development and cross-validation. Psychological Medicine, 24 (1), 145-153. doi: http://doi.org/10.1017/s003329170002691x

[24] Droś, J., Klimkowicz-Mrowiec, A. (2021). Current view on post-stroke dementia. Psychogeriatrics. doi: http://doi.org/10.1111/ psyg. 12666

[25] Hesseberg, K., Tangen, G. G., Pripp, A. H., Bergland, A. (2020). Associations between Cognition and Hand Function in Older People Diagnosed with Mild Cognitive Impairment or Dementia. Dementia and Geriatric Cognitive Disorders Extra, 10 (3), 195-204. doi: http://doi.org/10.1159/000510382

How to cite: Uwa-Agbonikhena, I., Gryb, V., Gerasymchuk, V., Kupnovytska-Sabadosh, M., Maksymchuk, L. (2021). Effect of the complex physical therapy on the upper extremity function, functional independence and cognition in post-stroke patients. EUREKA: Health Sciences, 3, 61-68. doi: http://doi.org/10.21303/2504-5679.2021.001768 